\title{
Parameters Calculation of Underground Cables Using MATLAB
}

\author{
Bin Sun \\ Hitachi ABB Power Grids, Raleigh, USA \\ Email: bins@clemson.edu
}

How to cite this paper: Sun, B. (2020) Parameters Calculation of Underground Cables Using MATLAB. Journal of Power and Energy Engineering, 8, 12-20. https://doi.org/10.4236/jpee.2020.811002

Received: October 14, 2020

Accepted: November 23, 2020

Published: November 26, 2020

Copyright $\odot 2020$ by author(s) and Scientific Research Publishing Inc. This work is licensed under the Creative Commons Attribution International License (CC BY 4.0).

http://creativecommons.org/licenses/by/4.0/

\begin{abstract}
With the development of technology, more and more options are available for insulated cables in power systems. Different cable construction types have different impedance, voltage drop and power losses. In order to efficiently design these cable systems and adequately model them for system analysis, engineers should be able to calculate the parameters of different types of cables. Although the methods of parameters calculation are very mature, few types of software are used to calculate all these parameters for different cables. The objective of this paper is to build a user-friendly software to calculate these parameters with greater flexibility even if the users are not familiar with the methods of estimating the parameters of different types of cables. A program that is used to calculate the parameters of different cross-sections of cables is built by combining the method of estimating these parameters with the graphical user interface (GUI). Using this program, users can input or choose any type of cables, and calculate the parameters they need. In the future, more functions can be added to the program and the code could be switched to python or C\# and develop a better GUI (Graphical User Interface).
\end{abstract}

\section{Keywords}

Carson Line Method, Tape Shield Cable, Concentric Neutral Cable

\section{Introduction}

Nowadays, more and more cables are used in power systems. To efficiently design these cable systems, engineers should be able to calculate the parameters of different types of cables. Although the methods, which include calculations of impedance matrix, power losses, and voltage drop, are very mature [1] [2], few types of software are used to calculate all these parameters for different cables [3]. There are some other cable data calculators [4] [5], but it's difficult for users 
to specify cables' cross-sections. The objective of this paper is to build a user-friendly software to calculate these parameters with greater flexibility even if the users are not familiar with the methods of estimating the parameters of different types of cables. To complete the program, it's necessary to know that two different types of cables are commonly used in the distribution system: concentric neutral cable and tape shield cable [1]. Before calculating the parameters of a cable, the composition of cables must be studied first. Generally, there are five levels of different materials. They are a copper conductor, EPR for fixation, insulation EPR, neutral copper conductors, and a jacket for physical protection as shown in Figure 1. However, for different types of cables, there are different conductor configurations.

For a common concentric neutral cable, the layer arrangement is shown in Figure 2. The inner layer is normally an aluminum conductor and the second layer is the EPR insulation. The circles around the insulation layer are several symmetrical copper conductors named neutral line. The outermost layer is a rubber jacket for physical protection. When the conductors are connected with a voltage source, the voltage distribution is shown in Figure 3.

For a standard tape shield cable, the layer arrangement is shown in Figure 4. The inner layer is a copper conductor and the second layer is the EPR insulation. The thin layer around the insulation layer is a copper shield that is normally grounded. The outermost layer is a rubber jacket for physical protection. When the conductors are connected with a voltage source, the voltage distribution is shown in Figure 5.

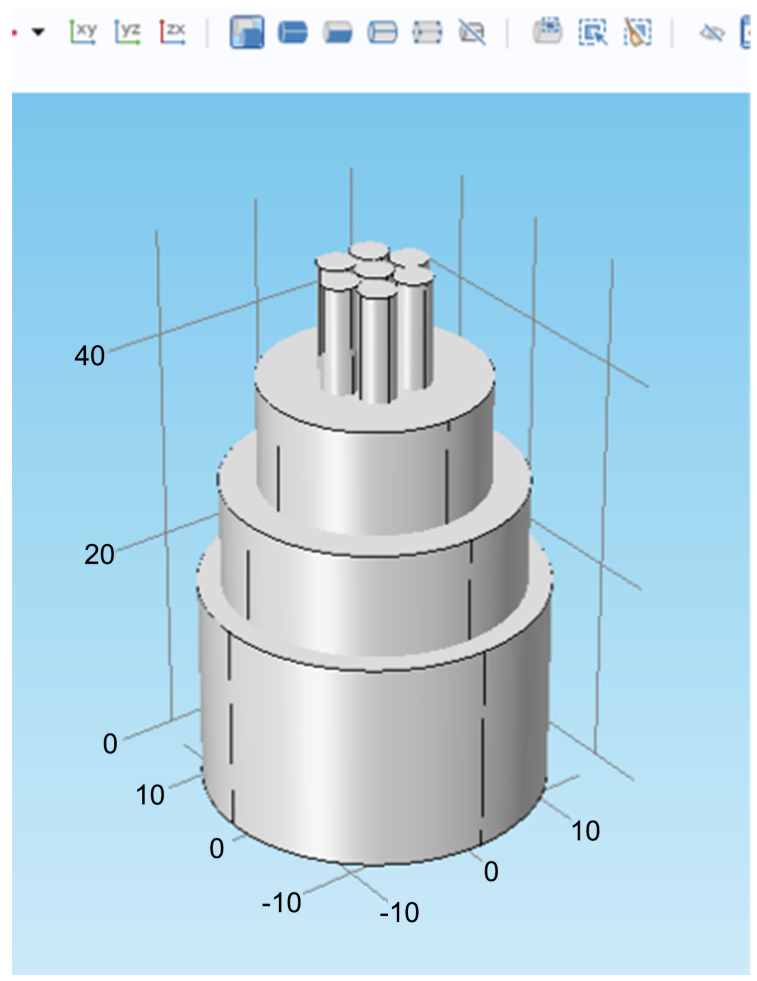

Figure 1. Common layer arrangement of a tape shield cable. 


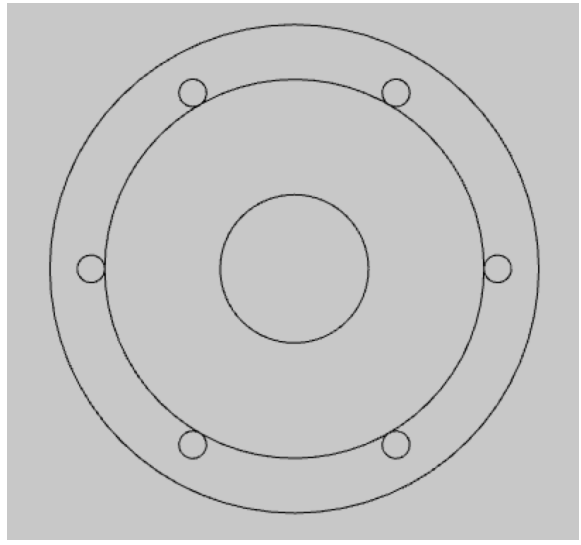

Figure 2. Cross section of a concentric neutral cable.

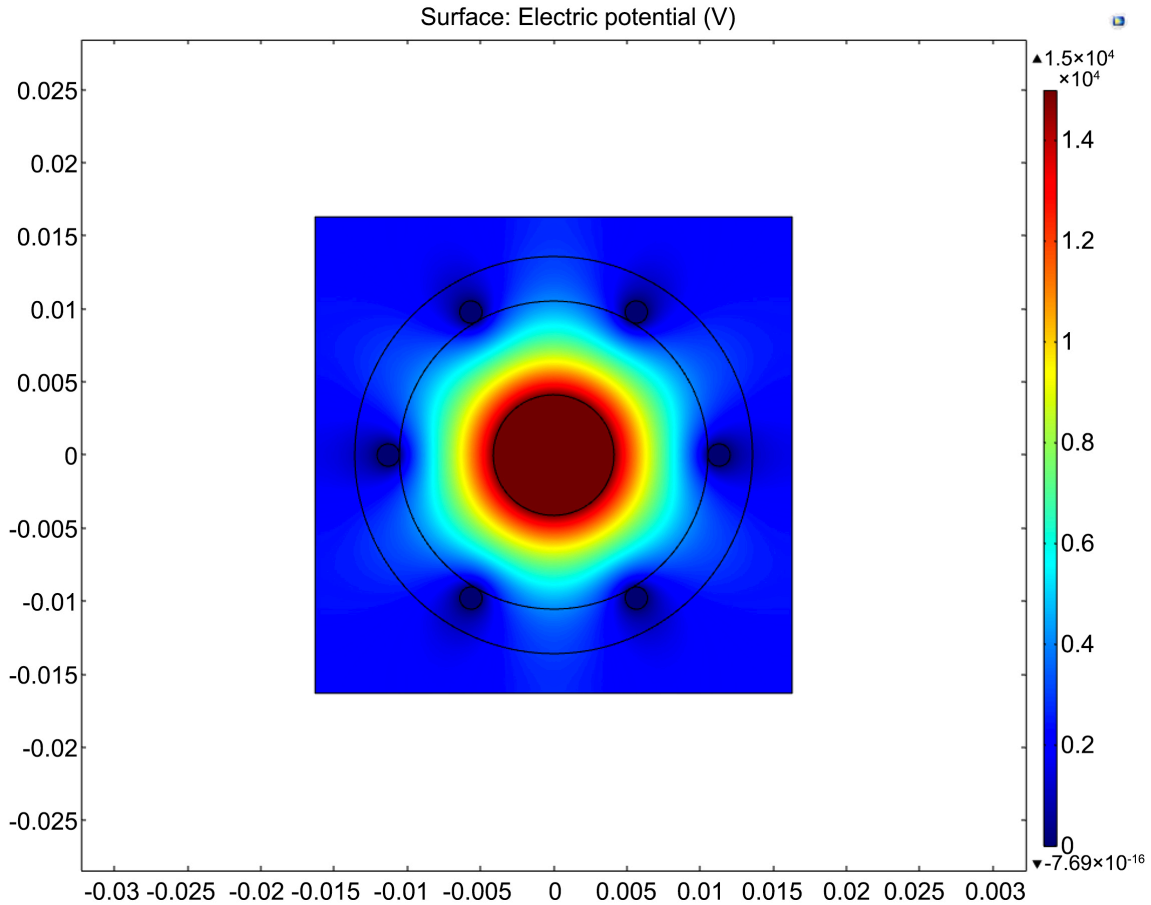

Figure 3. Voltage distribution of a concentric neutral cable.

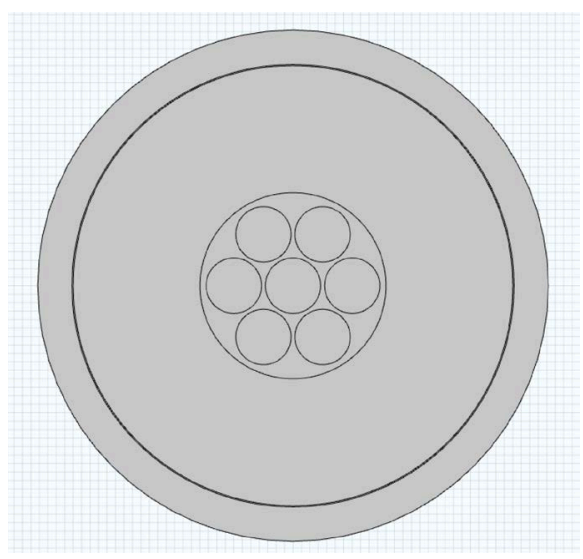

Figure 4. Cross-section of a tape shield cable. 


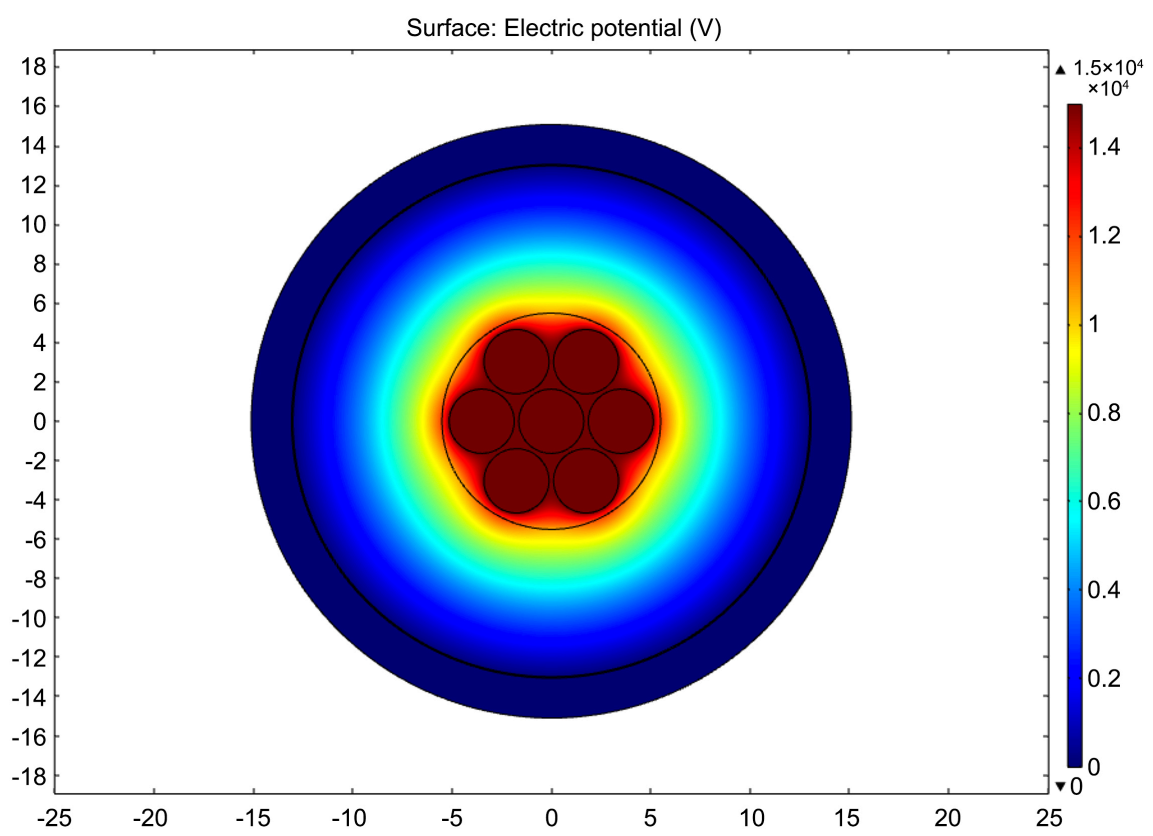

Figure 5. Voltage distribution of a tape shield cable.

\section{Method}

Carson line method is used to estimate the impedance matrix and calculate the parameters of these two types of cables [1]. For a concentric neutral cable, Equations (1)-(6) below are used to estimate the parameters [1].

$$
\begin{gathered}
Z_{a a}=\left(r_{a}+r_{d}\right)+j \omega k \ln \left(D_{e} / D_{s a}\right) / \text { mile } \\
Z_{a b}=r_{d}+j \omega k \ln \left(D_{e} / D_{a b}\right) / \text { mile } \\
\mathrm{GMR}=\sqrt[K]{\mathrm{GMR}_{s} * K * R^{K-1}} f t \\
R=\left(d_{o d}-d_{s}\right) / 2=\left(d_{o d}-d_{s}\right) / 2 * 12 f t \\
r_{c n}=r_{s} / K \Omega / \text { mile } \\
D_{i j 3}=\sqrt[K]{D_{n m}^{K}-R^{K}}
\end{gathered}
$$

where $D_{s a}$ is the conductor GMR in $f t, D_{e}$ is the earth GMR constant $=2790 \mathrm{ft}$ for average earth; $\omega k=0.12134 ; r_{a}$ is the resistance of the conductor in $\Omega /$ unit length; $r_{d}=0.09528 \Omega / \mathrm{mile}$, is the resistance of Carson line; $d_{o d}$ is the nominal diameter of the cable in inches; $d_{s}$ is the diameter of the neutral conductor in inches; $\mathrm{GMR}_{s}$ is the geometric mean radius of the neutral conductor in $f t, r_{s}$ is the resistance of neutral conductor in $\Omega /$ mile; $r_{d}$ is the earth constant resistance coefficient; $K$ is the number of concentric neutral strands; $R$ is the radius of a circle passing through the center of the concentric neutral strands; $r_{c n}$ is the equivalent resistance of the concentric neutral. These parameters are shown in Figure 6. Equation (1) and (2) are used to calculate self-impedance and mutual impedance separately.

For a tape shield cable, the parameters are shown in Figure 7. All equations are the same except Equations (3) and (5) [1]. 


$$
\begin{gathered}
\mathrm{GMR}_{\text {shield }}=\left(d_{s}-T / 1000\right) / 2 * 12 \text { in } f t \\
r_{\text {shield }}=7.9385 * 10^{8} \frac{P(\Omega * m)}{d_{s}(\text { inch }) * T(\text { m.inch })}
\end{gathered}
$$

Using the above equations combined with some other basic electrical equations, the impedance matrix of any cross-sections can be calculated. For example, the cross-section is shown in Figure 8.

Using Equation (1) and (2), a $13 \times 13$ impedance matrix could be developed with the corresponding conductor positions.

In order to simplify, number all the conductors:

Top Row: A1 = "1", C1 = "2", B1 = "3", B2 = “4", C2 = "5", A2 = "6".

Bottom Row: $\mathrm{A} 1$ = “7”, $\mathrm{C} 1$ = "8”, B1 = “9”, B2 = "10", C2 = "11", $\mathrm{A} 2$ = “12".

Ground: $\mathrm{G}=$ " 13 ".

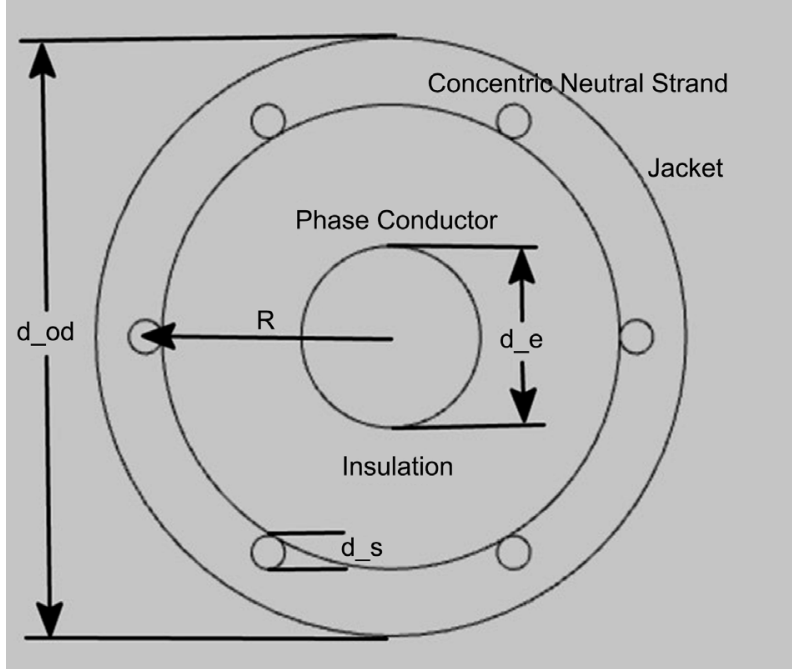

Figure 6. Parameters used in Carson line method for concentric neutral cable.

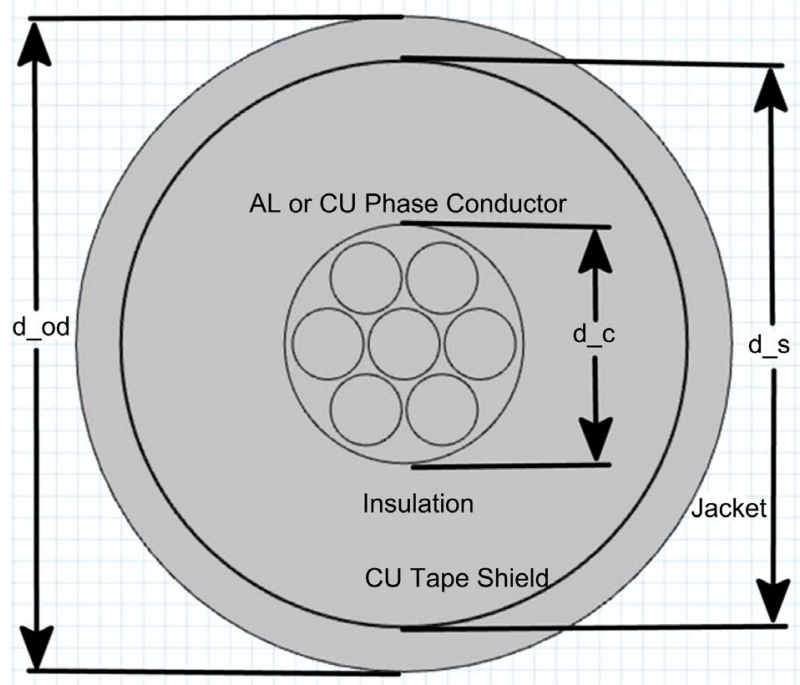

Figure 7. Parameters used in Carson line method for tape shield cable. 


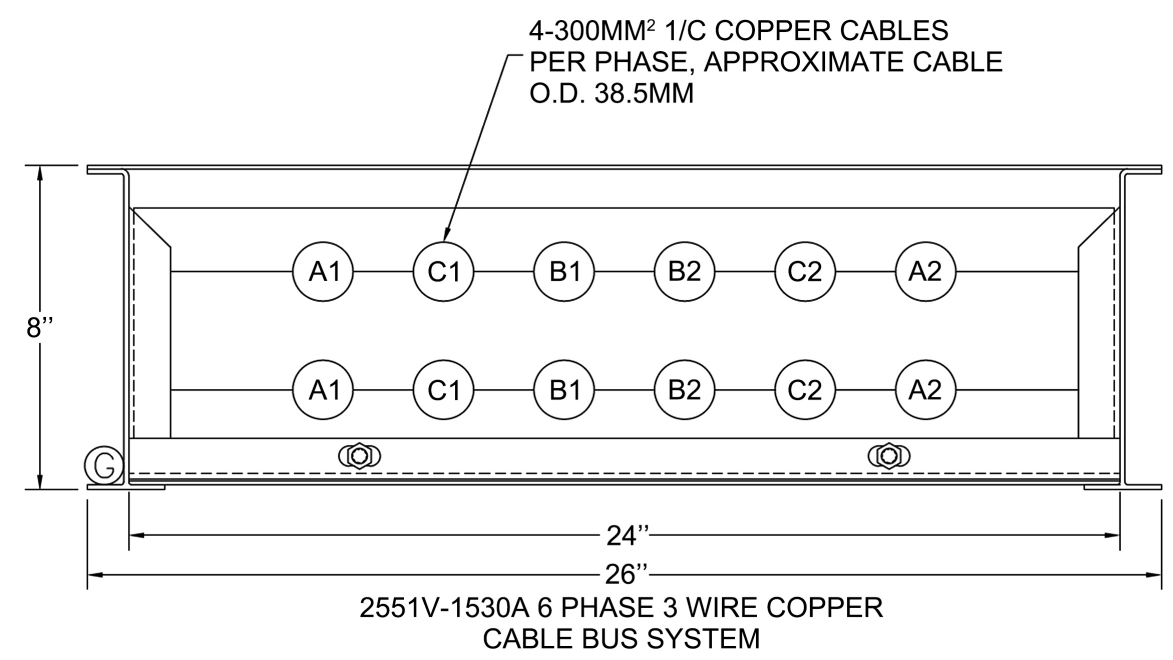

Figure 8. Sample cable arrangement.

The output impedance matrix is as follows:

$$
Z_{\text {abcg }}=\left[\begin{array}{cccc}
z_{1,1} & \cdots & z_{1,12} & z_{1,13} \\
\vdots & \ddots & \vdots & \vdots \\
z_{12,1} & \cdots & z_{12,12} & z_{12,13} \\
z_{13,1} & \cdots & z_{13,12} & z_{13,13}
\end{array}\right]_{13 \times 13}
$$

Because the 13th row and 13th column correspond to the ground conductor, the ground conductor can be eliminated by the equation:

$$
Z_{\mathrm{abc}}=\left[\begin{array}{ccc}
z_{1,1} & \cdots & z_{1,12} \\
\vdots & \ddots & \vdots \\
z_{12,1} & \cdots & z_{12,12}
\end{array}\right]_{12 \times 12} \quad-\left[\begin{array}{c}
z_{1,13} \\
\vdots \\
z_{12,13}
\end{array}\right] \frac{1}{z_{13,13}}\left[\begin{array}{lll}
z_{13,1} & \cdots & z_{13,12}
\end{array}\right]
$$

This equation is also called Kron Reduction [2].

Now the impedance matrix of the line is calculated. But the loads on the bus that is connected with the ground should be considered too. So the $Z_{\mathrm{abc}}$ matrix for the cable bus plus the load is calculated using

$$
Z_{\text {abc,total }}=\left[\begin{array}{ccc}
z_{1,1} & \cdots & z_{1,12} \\
\vdots & \ddots & \vdots \\
z_{12,1} & \cdots & z_{12,12}
\end{array}\right]_{12 \times 12}+\left[\begin{array}{ccc}
Z_{\text {load }, 1} & \cdots & 0 \\
\vdots & \ddots & \vdots \\
0 & \cdots & Z_{\text {load, }, 2}
\end{array}\right]
$$

Using the total impedance matrix established, the current flowing through the conductors can be calculated using Equation (11).

$$
\boldsymbol{V}_{\text {input }}=Z_{\text {abc,total }} \cdot \boldsymbol{I}
$$

where the current vector (I) has $12 \times 1$ complex numbers for the 12 conductors. Therefore, the input voltage vector $\left(V_{\text {input }}\right)$, total impedance matrix, and the current vector can be calculated as

$$
\boldsymbol{I}=Z_{\mathrm{abc}, \text { total }}^{-1} \cdot \boldsymbol{V}_{\text {input }}
$$

where the input voltages are described in Equation (14) if the system is balanced: 


$$
V_{A 1}=V_{A 2}=V_{A 3}=V_{A 4}=\frac{V_{\text {rated }}}{\sqrt{3}} \angle 0^{\circ} \quad V_{B 1}=V_{B 2}=V_{B 3}=V_{B 4}=\frac{V_{\text {rated }}}{\sqrt{3}} \angle-120^{\circ} \quad V_{\text {input }}=\left[\begin{array}{c}
V_{A 1} \\
V_{C 1} \\
V_{B 1} \\
V_{B 2} \\
V_{C 2} \\
V_{A 2} \\
V_{A 3} \\
V_{C 3} \\
V_{B 3} \\
V_{B 4} \\
V_{C 4} \\
V_{A 4}
\end{array}\right]
$$

If there are multiple neutral lines, the conventional method is to reduce them into one equivalent neutral line, which follows Equation (15).

$$
D_{s}=\sqrt[n^{2}]{\left(D_{a a} D_{a b} \cdots D_{a n}\right)\left(D_{b a} D_{b b} \cdots D_{b n}\right) \cdots\left(D_{n a} D_{n b} \cdots D_{n n}\right)}
$$

where $D_{i j}$ is the distance between neutral $i$ and neutral $j$. If $i=j, D_{i j}$ means GMR; $n$ means $n$-strand bundle neutral.

\section{Results}

Using Carson line method, a software that is used to calculate the parameters of two types of cables for different cross-sections was developed. Using this program, users can input or choose some initial values, and calculate the parameters they need, such as impedance matrix, voltage drop and power loss.

In this program, there are 26 preloaded cross-sections that are ready to be used. If they are not enough, this program allows the user to specify the locations of all individual cables. When the user inputs the line to line voltage, current, power factor of the load, ambient temperature, conductor type, cable bus length, cable's outer diameter, conductor size, diameter of ground, size of ground and specified cross-section, the program will look up standard tables of different cables and obtain related parameters, such as conductor resistance and its diameter. Then it will use the Carson line method and cable equations to calculate the final impedance matrix to be used for power losses and voltage drop calculations. The main process of the MATLAB code is shown in Figure 9.

Based on the MATLAB impedance matrix calculation code, the graphical user interface (GUI) of this program was built, and the sample calculation results were displayed, as shown in Figure 10. Using this GUI, users can choose and input initial values before easily calculating the impedance matrix to be used for different calculations.

\section{Conclusion}

In recent years, more and more electrical utilities are using cables to distribute electric power to their customers. However, few types of software are developed 


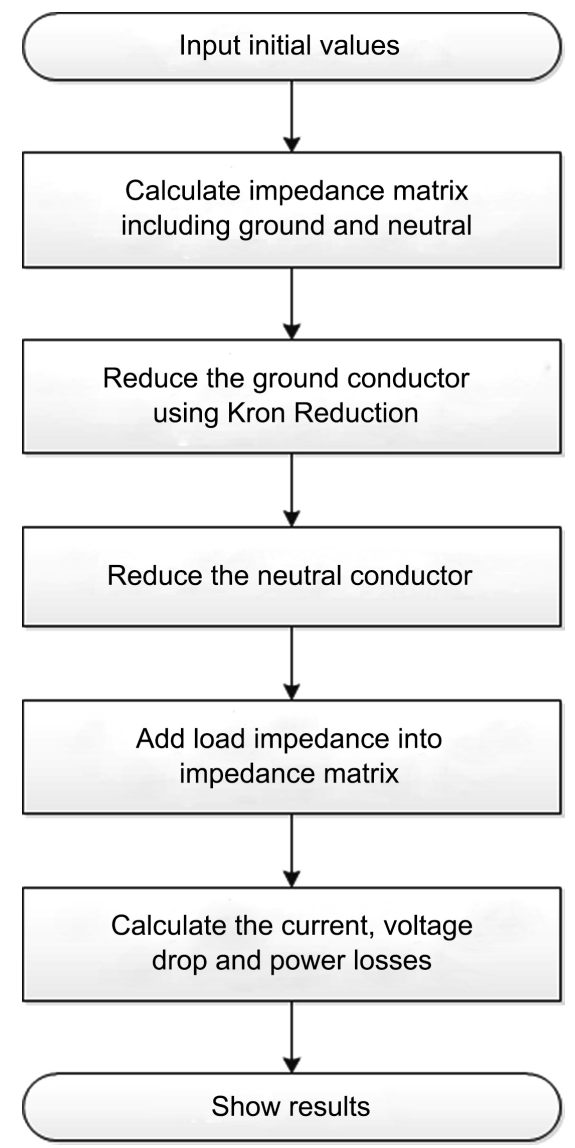

Figure 9. Steps of MATLAB code.

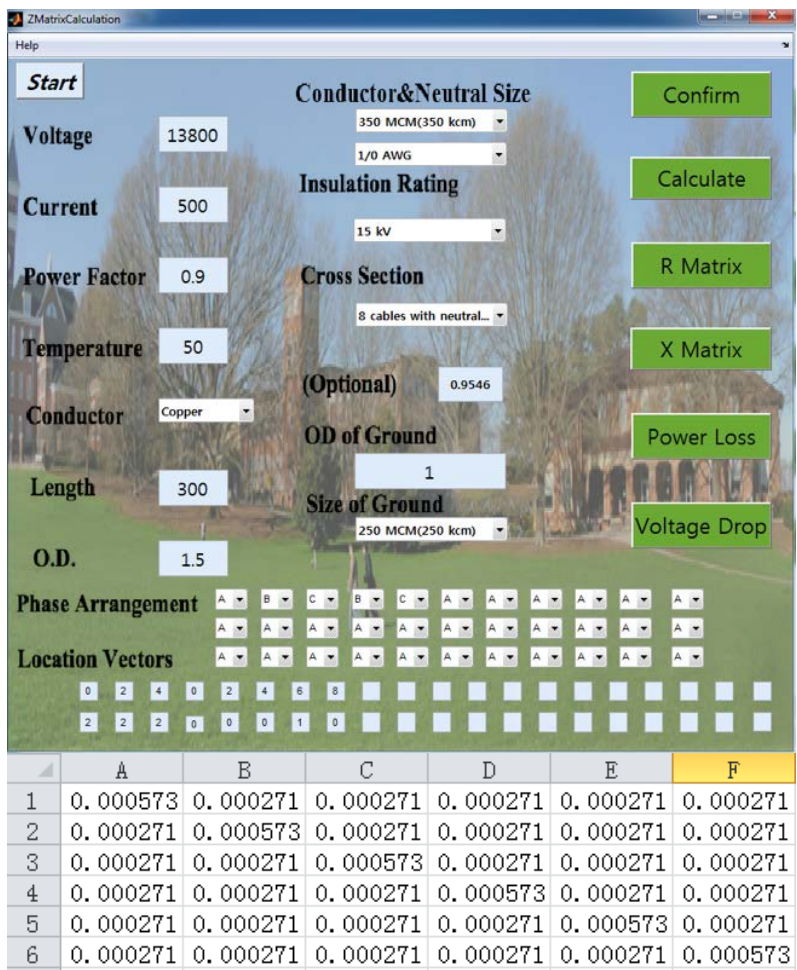

Figure 10. GUI interface and impedance matrix results. 
to calculate parameters for different cables. In this paper, a MATLAB-based program is built to calculate parameters of different types of cables including impedance matrix, power losses, and voltage drop. Users can input and select some initial values and easily calculate the parameters they need using the developed user-friendly software. With these parameters, power utilities can realize the conditions of cables and predict their voltage drop and power losses, and select the best type of cables they need.

\section{Conflicts of Interest}

The author declares no conflicts of interest regarding the publication of this paper.

\section{References}

[1] Gonen, T. (2013) Electric Power Distribution Engineering. Third Edition, CRC Press, Boca Raton.

[2] Grainger, J.J. and Stevenson, W.D. (1994) Power System Analysis. McGraw-Hill Book Co., New York.

[3] CYME International-CYMCAP. http://www.cyme.com/software/cymcap/

[4] Calculation of Cable Data. http://nepsi.com/resources/calculators/calculation-of-cable-data.htm

[5] General Cable Tools. https://www.generalcable.com/eu/en/information-center/tools-applications/gc-applow-voltage 